\title{
Téoros
}

Revue de recherche en tourisme

\section{Cogestion adaptative des parcs du Nunavik dans un contexte de changements climatiques}

\author{
Yanick Gendreau, Catherine A. Gagnon, Dominique Berteaux et Fanie Pelletier
}

Volume 31, numéro 1, 2012

Parcs du Nunavik

URI : https://id.erudit.org/iderudit/1020710ar

DOI : https://doi.org/10.7202/1020710ar

Aller au sommaire du numéro

Éditeur(s)

Université du Québec à Montréal

ISSN

0712-8657 (imprimé)

1923-2705 (numérique)

Découvrir la revue

Citer cet article

Gendreau, Y., Gagnon, C. A., Berteaux, D. \& Pelletier, F. (2012). Cogestion adaptative des parcs du Nunavik dans un contexte de changements climatiques. Téoros, 31(1), 61-71. https://doi.org/10.7202/1020710ar
Résumé de l'article

Les changements climatiques auront des répercussions sur les systèmes socioécologiques au sein desquels les Inuits évoluent. Les parcs sont identifiés comme un des outils pour faciliter l'adaptation des populations locales aux changements climatiques en permettant, entre autres, de maintenir des biens et services environnementaux. Pour que les parcs puissent assurer ce rôle au Nunavik, nous proposons ici un modèle de cogestion adaptative, favorisant l'implication des populations locales. Ainsi, le modèle présenté est innovateur, car il tient compte de la problématique de gestion des parcs dans le contexte des changements climatiques, mais aussi de la flexibilité nécessaire à une implication accrue des Inuits au niveau de la gestion des parcs. 


\title{
Cogestion adaptative des parcs du Nunavik dans un contexte de changements climatiques
}

\author{
Yanick GENDREAU \\ Doctorant \\ Chaire de recherche du Canada en biodiversité nordique \\ Département de biologie, Université du Québec à Rimouski \\ yanick.gendreau@uqar.qc.ca \\ Catherine A. GAGNON \\ Doctorante \\ Chaire de recherche du Canada en biodiversité nordique \\ Département de biologie, Université du Québec à Rimouski \\ catherine-alexandra.gagnon@uqar.qc.ca
}

\author{
Dominique BERTEAUX, Ph.D. \\ Professeur \\ Chaire de recherche du Canada en biodiversité nordique \\ Département de biologie, Université du Québec à Rimouski \\ dominique_berteaux@uqar.qc.ca \\ Fanie PELLETIER, Ph.D. \\ Professeure agrégée, Chaire de recherche du Canada \\ en démographie évolutive et conservation \\ Département de biologie, Université de Sherbrooke \\ fanie.pelletier@usherbrooke.ca
}

\begin{abstract}
RÉSUMÉ: Les changements climatiques auront des répercussions sur les systèmes socioécologiques au sein desquels les Inuits évoluent. Les parcs sont identifiés comme un des outils pour faciliter l'adaptation des populations locales aux changements climatiques en permettant, entre autres, de maintenir des biens et services environnementaux. Pour que les parcs puissent assurer ce rôle au Nunavik, nous proposons ici un modèle de cogestion adaptative, favorisant l'implication des populations locales. Ainsi, le modèle présenté est innovateur, car il tient compte de la problématique de gestion des parcs dans le contexte des changements climatiques, mais aussi de la flexibilité nécessaire à une implication accrue des Inuits au niveau de la gestion des parcs.
\end{abstract}

Mots-clés: Changements climatiques, cogestion adaptative, Nunavik, parc.

Il est maintenant bien connu que les changements climatiques auront des impacts importants sur la biodiversité, et ce, à l'échelle planétaire (Thomas et al., 2004). Ces effets seront encore plus marqués pour les régions nordiques, tel le Nunavik, et les parcs qui s'y trouvent (Callaghan et al., 2004). Conséquemment, les systèmes socioécologiques qui dépendent des biens et services que procurent les écosystèmes (par exemple : services de régulation, d'approvisionnement et socioculturels) s'en trouveront perturbés (Dudley et al., 2010). Dans ce contexte, les parcs font aussi partie des outils disponibles pour réduire les impacts négatifs des changements climatiques sur les systèmes socioécologiques (Welch, 2005). En effet, l'état actuel des connaissances démontre que les écosystèmes et les espèces qui résident dans les parcs sont moins sensibles aux changements climatiques (plus résilients) que ceux qui évoluent en territoires non protégés. Ceci s'explique par le fait qu'ils sont généralement moins exposés à d'autres sources de stress (par exemple : perte d'habitat, surexploitation, pollution, etc.) qui agissent souvent en synergie avec les changements climatiques (Hannah et al., 2007; Dudley et al., 2010). De plus, les parcs peuvent faciliter l'adaptation des populations locales aux changements climatiques et augmenter la résilience des systèmes socioécologiques en assurant le maintien des biens et services écologiques, en jouant un rôle tampon sur le climat local, et en atténuant les risques et les impacts des événements climatiques extrêmes (Dudley et al., 2010).

En vertu de ces «nouvelles» utilités que l'on attribue aux parcs, mais aussi parce que les connaissances des impacts des changements climatiques sur ces derniers sont incomplètes, il est nécessaire que les gestionnaires de parcs appliquent le principe de précaution et se dotent d'un nouveau modèle de gestion dynamique que l'on nomme gestion adaptative (Welch, 2005). La gestion adaptative se résume brièvement comme un mode de gestion mettant à profit les leçons tirées de pratiques antérieures pour améliorer la gestion actuelle. Cette approche a d'abord été développée pour gérer des problématiques liées à l'exploitation des ressources naturelles impliquant plusieurs enjeux (humains, économiques et écologiques). Toutefois, cette notion d'amélioration constante des mesures de gestion peut aussi s'appliquer aux mécanismes de cogestion (considérer plus d'un intervenant dans le modèle de gestion et impliquer les populations locales) de façon à ce qu'ils soient eux-mêmes soumis à de fréquentes évaluations et améliorations (processus dynamique). Nous parlerons alors de cogestion adaptative. Considérant les incertitudes liées aux changements climatiques et à l'établissement d'organisations de cogestion, le concept de cogestion adaptative semble fort approprié pour la gestion des parcs du Nunavik. 


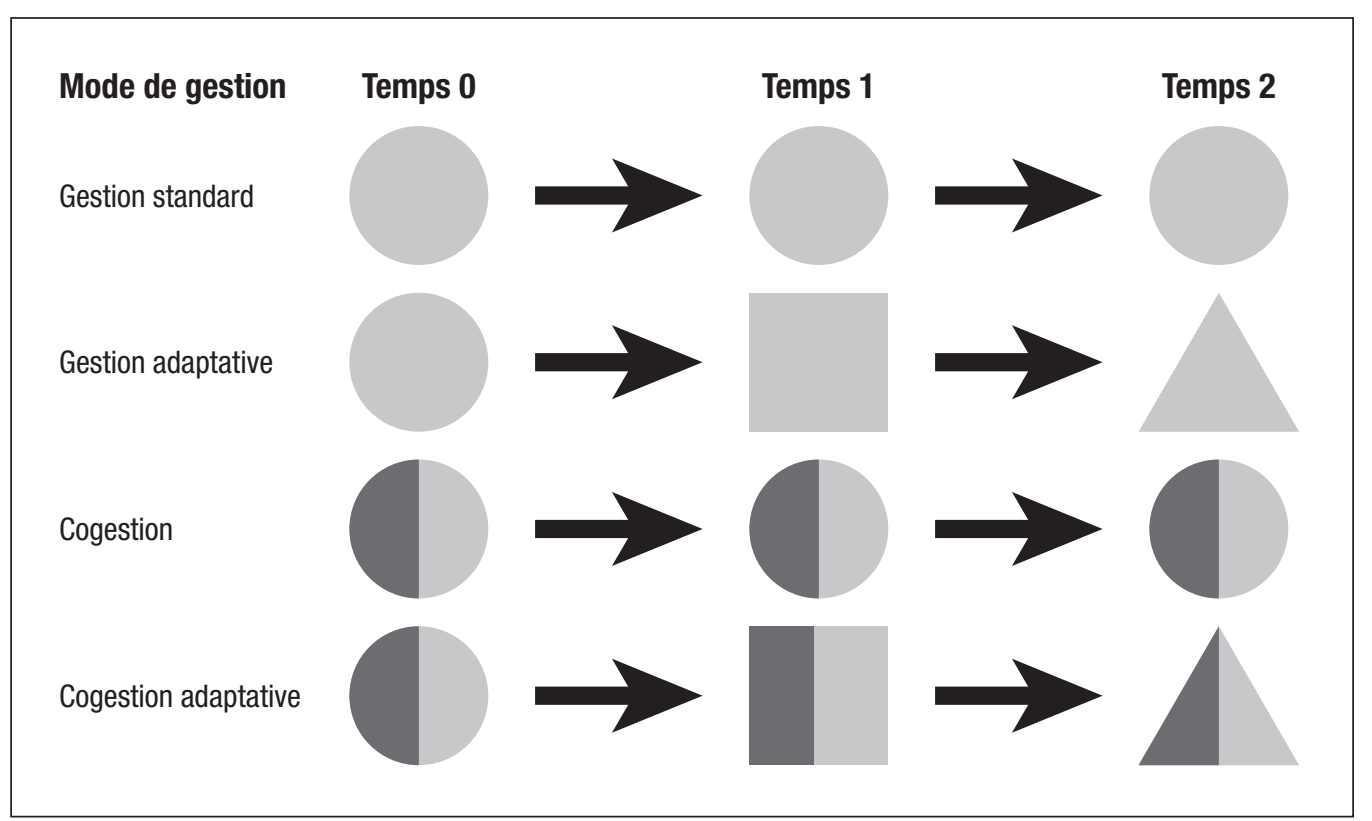

\author{
ILLUSTRATION 1 : Présentation \\ des différents modes de gestion \\ des ressources naturelles \\ (incluant les parcs) et de leur \\ évolution dans le temps. Les \\ différentes formes illustrent \\ les changements dans les \\ mesures de gestion et dans \\ les mécanismes de cogestion \\ au fil du temps (adaptatif). Les \\ différentes teintes de gris et \\ leurs proportions respectives \\ illustrent les différents acteurs \\ impliqués dans la cogestion (par \\ exemple, locaux : gris foncé; \\ gouvernementaux : gris pâle) \\ (source : compilation des auteurs).
}

L'objectif de cet article est de présenter une méthode novatrice de gestion des parcs, à partir de l'exemple du Nunavik, qui tienne compte de la problématique des changements climatiques, mais aussi de la flexibilité nécessaire à une implication accrue des Inuits au niveau de la gestion des parcs. Le tout doit concilier le mandat de conservation et de développement touristique auxquels l'Administration régionale Kativik (ARK) doit répondre. Le danger de suggérer un mode de gestion est toujours de paraître normatif et de sembler vouloir faire dire encore une fois aux Inuits ce qui est mieux pour eux. Notre intention est tout à fait contraire puisque la cogestion adaptative que nous proposons vise à donner du pouvoir aux personnes vivant dans ou près des parcs. Ainsi, le modèle de cogestion adaptative que nous proposons comporte cinq étapes : 1) l'élaboration d'un plan régional;2) l'élaboration du plan de gestion pour chaque parc; 3) l'application du plan de gestion à chaque parc; 4) la surveillance socioenvironnementale et l'acquisition de connaissances; 5) la synthèse, analyses et modification des objectifs. À ce jour, un tel modèle de cogestion des parcs nordiques dans le contexte des changements climatiques n'a jamais été proposé et utilisé, autant au niveau provincial, national, qu'international.

\section{Méthodologie}

\section{Origine de la démarche}

Le modèle de cogestion adaptative que nous proposons peut se décliner en deux composantes. Une des composantes est les changements climatiques et l'autre est la cogestion adaptative. La structure même du modèle est inspirée d'un rapport intitulé Impacts des changements climatiques sur la biodiversité du Québec : Aires protégées produit pour le ministère du Développement durable, de l'Environnement et des Parcs du Québec et le Centre de la science de la biodiversité du Québec. Ce modèle a été développé par Gendreau et Pelletier, coauteurs du présent article. Toutefois, ici, contrairement au rapport de Chambers et al. (2010), les étapes du modèle sont mieux définies et sont présentées sous forme d'illustration en boucle, basée sur le modèle de la gestion adaptative (Sainsbury et al., 2000). Le contenu de chacune des étapes est lui-même inspiré de la convergence que l'on retrouve dans la littérature scientifique qui traite des effets des changements climatiques sur les aires protégées (dont les parcs) (Welch, 2005; Mawdsley et al., 2009; Dudley et al., 2010).

En nous basant sur ce modèle de gestion adaptative des parcs dans le contexte des changements climatiques, nous avons intégré les connaissances et l'expérience acquise par Gagnon et Berteaux (2006) lors de leurs travaux sur l'inclusion du savoir inuit dans la gestion des parcs nationaux du Canada au Nunavut pour en faire un modèle de cogestion adaptative unique. Pour cette tâche, nous nous sommes inspirés de ce qui est présentement fait dans le domaine de la cogestion des parcs au Nunavik. La résultante est un modèle de gestion adaptative en cogestion avec les Inuits, axé sur le développement de liens de confiance entre les différents partis impliqués, et au sein duquel les Inuits sont des partenaires à part entière. Nous voyons ainsi la cogestion non pas comme un processus consultatif, mais comme un mode de gouvernance dans lequel les Inuits ont des pouvoirs décisionnels. Nous suggérons aussi dans ce modèle que les connaissances des Inuits prennent une place importante lors du processus de cueillette d'informations sur les parcs visés.

\section{Définition des termes :}

\section{de la gestion à la cogestion adaptative}

Prendre des décisions à partir d'informations limitées ou fragmentaires est chose commune en gestion et conservation des ressources naturelles (Welch, 2005; McCarthy et Possingham, 2007). Cette réalité est en grande partie due au fait que la recherche appliquée en écologie est une entreprise difficile : les expériences sont longues et les réplicas peu nombreux 


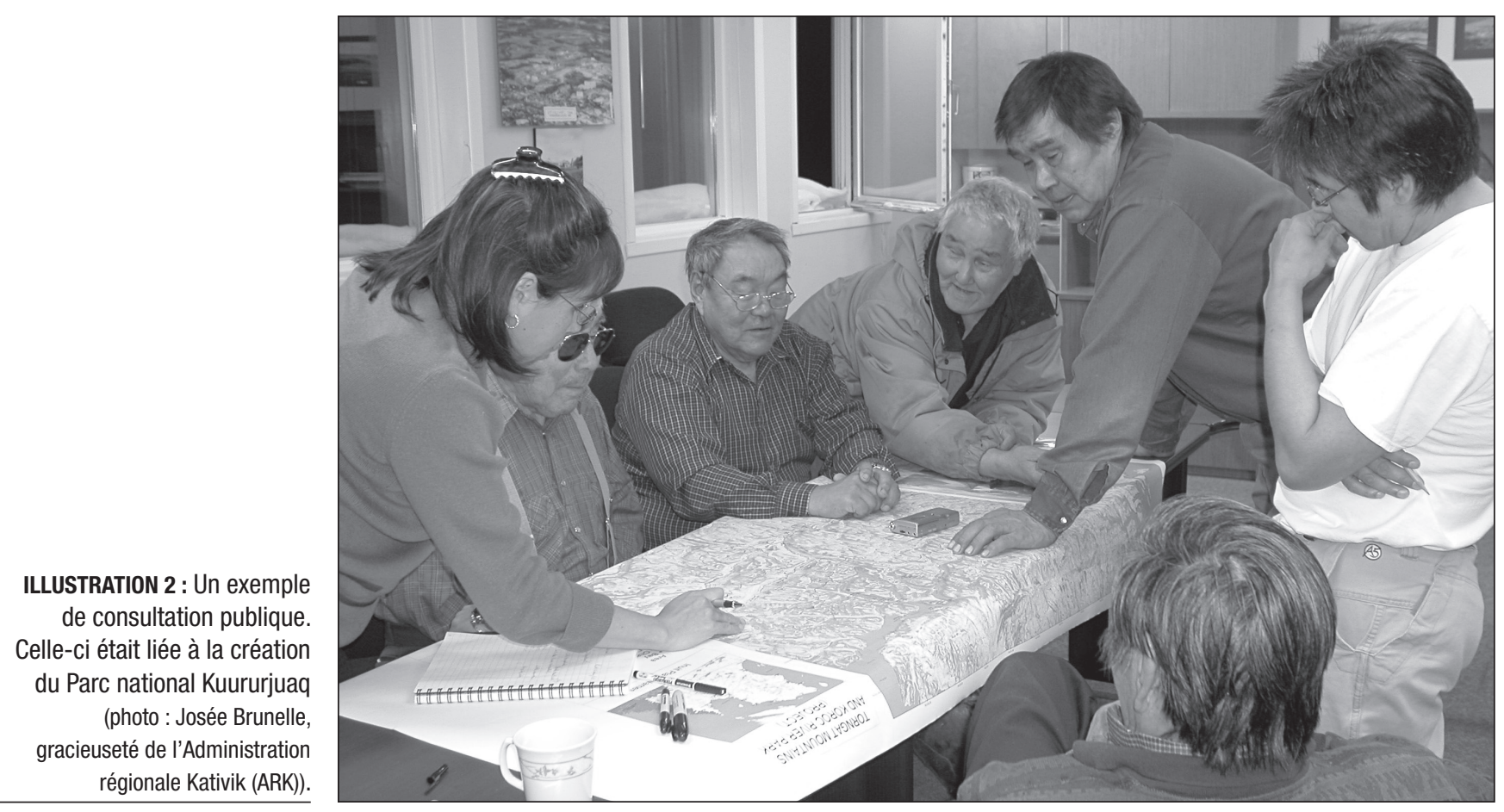

(Hilborn et Ludwig, 1993). Dans le cas des changements climatiques par exemple, il est impossible de manipuler le climat et de faire des expériences en milieu contrôlé (Krebs et Berteaux, 2006). Il est donc impossible de confirmer avec certitude les conséquences que ces changements auront sur les écosystèmes et les espèces, ce qui complique les prises de décisions en matière de gestion environnementale (Krebs et Berteaux, 2006). De plus, les avancées scientifiques des dernières décennies tendent à montrer que les écosystèmes soumis à un stress ne changent pas graduellement, mais plutôt de manière brusque et imprévisible (Holling, 1996). C'est afin de tenir compte de ces incertitudes et de la nature imprévisible des écosystèmes que la méthode de la gestion adaptative a été proposée (Holling, 1978).

Selon l'Évaluation des écosystèmes pour le millénaire (EM, 2005 : 599), la gestion adaptative se définit comme un "processus systématique d'amélioration continue des politiques et pratiques de gestion qui se base sur les leçons tirées des résultats de politiques et pratiques antérieures» (traduction libre des auteurs (TLDA)). En termes pratiques, il s'agit d'un mode de gestion dynamique où les résultats sont suivis, quantifiés et analysés (généralement par une méthode scientifique). Au fur et à mesure que de nouvelles informations sont disponibles, les décisions de gestion sont modifiées ou adaptées afin de poursuivre les objectifs fixés, et ainsi de suite (Walters, 1986; Welch, 2005; voir illustration 1). La gestion adaptative est donc basée sur un apprentissage continu et considère, en quelque sorte, les politiques de gestion comme des expériences à partir desquelles les gestionnaires peuvent en apprendre davantage sur les écosystèmes qu'ils gèrent (Berkes et Folke, 1998). Or, afin de rendre ce processus légitime et acceptable par les populations locales, plusieurs auteurs ont prôné l'implication des acteurs locaux dans les processus de génération de connaissances et d'apprentissages propres à la gestion adaptative (Berkes et Folke, 1998; Armitage et al., 2007b). Ceci devait faire converger la gestion adaptative vers des modes de gestion plus collaboratifs, telle la cogestion.

Autrefois essentiellement réservée aux experts scientifiques, la gestion des ressources naturelles s'est ouverte, depuis une trentaine d'années, à l'implication de différents acteurs locaux, tels les Autochtones. Cette ouverture a surtout découlé des luttes politiques entre Autochtones et non-Autochtones. Tout savoir est source de pouvoir et la prise en compte des savoirs autochtones dans les décisions était, pour ceux-ci, une des meilleures façons de reprendre un peu de contrôle sur ce qui se passait chez eux. Ainsi est apparu, dans les années 1990, le concept de cogestion, symbolisant ce désir d'accroître le partage des responsabilités quant à la gestion des ressources (voir illustration 1). Berkes et al. (1991) définissent la cogestion comme «le partage du pouvoir et des responsabilités entre le gouvernement et les utilisateurs locaux des ressources" (TLDA). En d'autres termes, la cogestion réfère à « une variété d'arrangements, avec différents degrés de partage du pouvoir, visant la prise de décisions conjointes, entre l'État et les communautés (ou groupes d'intérêts), concernant une série de ressources ou une région" (Berkes, 2009, TLDA). En pratique, la cogestion se concrétise différemment selon les situations, sur un continuum partant de la simple consultation publique (illustration 2) jusqu'à l'établissement de partenariats formels (Borrini-Feyerabend et al., 2004). L'État et les communautés impliquées dans une situation de cogestion sont rarement deux unités monolithiques, l'État représentant plutôt une variété d'agences gouvernementales, et les communautés, une panoplie d'intérêts locaux (Carlsson et Berkes, 2005). 
La pratique de la cogestion n'est donc pas une entreprise simple. Selon plusieurs auteurs, elle présente néanmoins plusieurs bénéfices potentiels, tels que la démocratisation de la prise de décisions, la mise en place de mécanismes pour la résolution de conflits et une gouvernance plus efficace et plus équitable (Armitage et al., 2007a). La cogestion peut aussi améliorer la prise de données, la protection des ressources, l'intégration de différents types de savoirs (par exemple : scientifiques et autochtones; voir encadré 1) et le respect des lois environnementales (Armitage et al., 2007a). Par contre, d'autres auteurs sont sceptiques quant au succès de ce mode de gestion, mentionnant le manque d'évidences sur les retombées positives réelles de la cogestion (Nadasdy, 2003; Plummer et FitzGibbon, 2004). Nadasdy (1999, 2003) va jusqu'à noter que la cogestion peut maintenir le déséquilibre du pouvoir existant entre l'État et les populations autochtones. Une solution potentielle pour rééquilibrer les pouvoirs consiste à voir la cogestion non pas comme un arrangement fixe et statique, mais plutôt comme un processus en constante évolution, impliquant bon nombre de délibérations (Carlsson et Berkes, 2005). Chaque partenariat de cogestion doit ainsi inclure une évaluation (ou autoévaluation) périodique qui analyse ses résultats au niveau institutionnel (par exemple, en termes d'équilibre du pouvoir) de l'état des écosystèmes gérés et des conditions de subsistance des populations locales impliquées (Plummer et Armitage, 2007). Autrement dit, le concept de cogestion devrait inclure la notion d'amélioration constante des politiques et des pratiques propres à la gestion adaptative pour former ce que l'on nomme un mode de cogestion adaptative (Armitage et al., 2007b).

Le concept de cogestion adaptative a fait surface dans la littérature sur la gestion des ressources naturelles au cours de la dernière décennie, découlant de la rencontre naturelle

\section{ENCADRÉ 1: PRINCIPAUX TYPES DE SAVOIRS UTILISÉS POUR LA GESTION DES PARCS}

\section{Savoirs scientifiques : Savoirs codifiés qui constituent la norme de référence pour expliquer la structure et le fonctionnement des écosystèmes. Ils sont à la base des conventions internationales comme la Convention sur la diversité biologique.}

Savoirs locaux (autochtones, paysans, etc.) : Savoirs non codifiés et souvent transmis oralement et par la pratique. Ils sont constitués d'observations, de croyances et de pratiques.

Savoirs savants naturalistes : lls sont intermédiaires entre les deux catégories ci-dessus. Ils sont codifiés et se transmettent par écrit, mais ne sont pas acquis par des méthodes scientifiques. Exemple : savoirs échangés au sein des groupes d'ornithologues.

Savoirs des gestionnaires des parcs : Savoirs composites acquis à partir des savoirs mentionnés ci-dessus et ajustés au contexte local grâce à l'expérience de terrain. Dans un contexte de prise de décision rapide et de manque de moyens pour approfondir chacun des savoirs ci-dessus, les décisions de gestion reposent souvent sur ces savoirs.

Source : Adapté de Prévot-Julliard et al. (2010 : 152). des concepts de gestion adaptative et de cogestion. Le concept de cogestion adaptative se définit comme «un processus par lequel les arrangements institutionnels et le savoir écologique sont testés et révisés, via un processus dynamique, continu et auto-organisé d'apprentissage par la pratique» (Folke et al., 2002, TLDA; voir illustration 1). La cogestion adaptative est donc une approche multidisciplinaire qui reconnaît que les systèmes humains (incluant les activités touristiques) et écologiques sont étroitement liés et doivent être vus comme des systèmes socioécologiques. Elle vise ultimement à favoriser la durabilité de ces systèmes (Armitage et al., 2007a). Pour atteindre cet objectif, elle fait usage d'éléments clés tels : l'utilisation de différents savoirs (tant scientifiques que locaux; voir encadré 1), le partage véritable du pouvoir, la construction de liens de confiance et de respect entre les acteurs impliqués et un apprentissage itératif mettant l'accent sur la flexibilité et l'adaptabilité. Pour ce faire, il est nécessaire de mettre en place des institutions regroupant plusieurs niveaux de gouvernance, et de renforcer la capacité de développement chez les acteurs locaux et gouvernementaux (Berkes et al., 2007). En pratique, la cogestion adaptative pourra prendre plusieurs formes, selon les situations. Nous proposons maintenant l'ébauche d'un modèle de cogestion adaptative spécifique à la situation des parcs du Nunavik et tenant compte de deux aspects fondamentaux de leur gestion : la problématique des changements climatiques et l'implication des Inuits.

Dans le passé, l'acquisition de connaissances, la gestion et la surveillance environnementale des parcs ont été essentiellement basées sur les méthodes et connaissances scientifiques, ainsi que sur l'expérience et les connaissances pratiques des gestionnaires. Toutefois, les recherches des dernières décennies ont démontré que la participation des Autochtones ou autres acteurs locaux à la recherche, à la gestion et au suivi environnemental peut être hautement bénéfique (Berkes, 1999, 2009; Wagner et Fernandez-Gimenez, 2008; Gagnon et Berteaux, 2009). De par les liens étroits qu'ils entretiennent avec leur environnement, les Autochtones du nord sont très bien placés pour observer certains des changements que subit leur territoire tant au niveau social qu'environnemental (Riedlinger et Berkes, 2001; Krupnik et Jolly, 2002; Huntington et al., 2004). Ils sont aussi les mieux placés pour exprimer ce qu'ils désirent par rapport à ces territoires. Ils possèdent enfin des connaissances sur les espèces et écosystèmes qui les entourent et peuvent ainsi fournir des observations et perspectives complémentaires aux données scientifiques (Gagnon et Berteaux, 2009).

Nous ne prétendons pas que ce modèle de cogestion adaptative soit définitif, immuable et qu'il devrait constituer une norme. Au contraire, un modèle de cogestion adaptative pour un système socioécologique exige : 1) que les objectifs fixés, tant pour les aspects sociaux qu'écologiques, soient suivis et analysés tout au long du processus; et 2) la participation des groupes d'utilisateurs (ici les Inuits) et la réévaluation périodique du rôle de ces derniers. Nous considérons que le modèle que nous proposons devrait servir de point de départ à l'implantation d'un modèle de cogestion adaptative pour les parcs du Nunavik dans le contexte des changements climatiques. Il comprend plusieurs étapes que voici. 


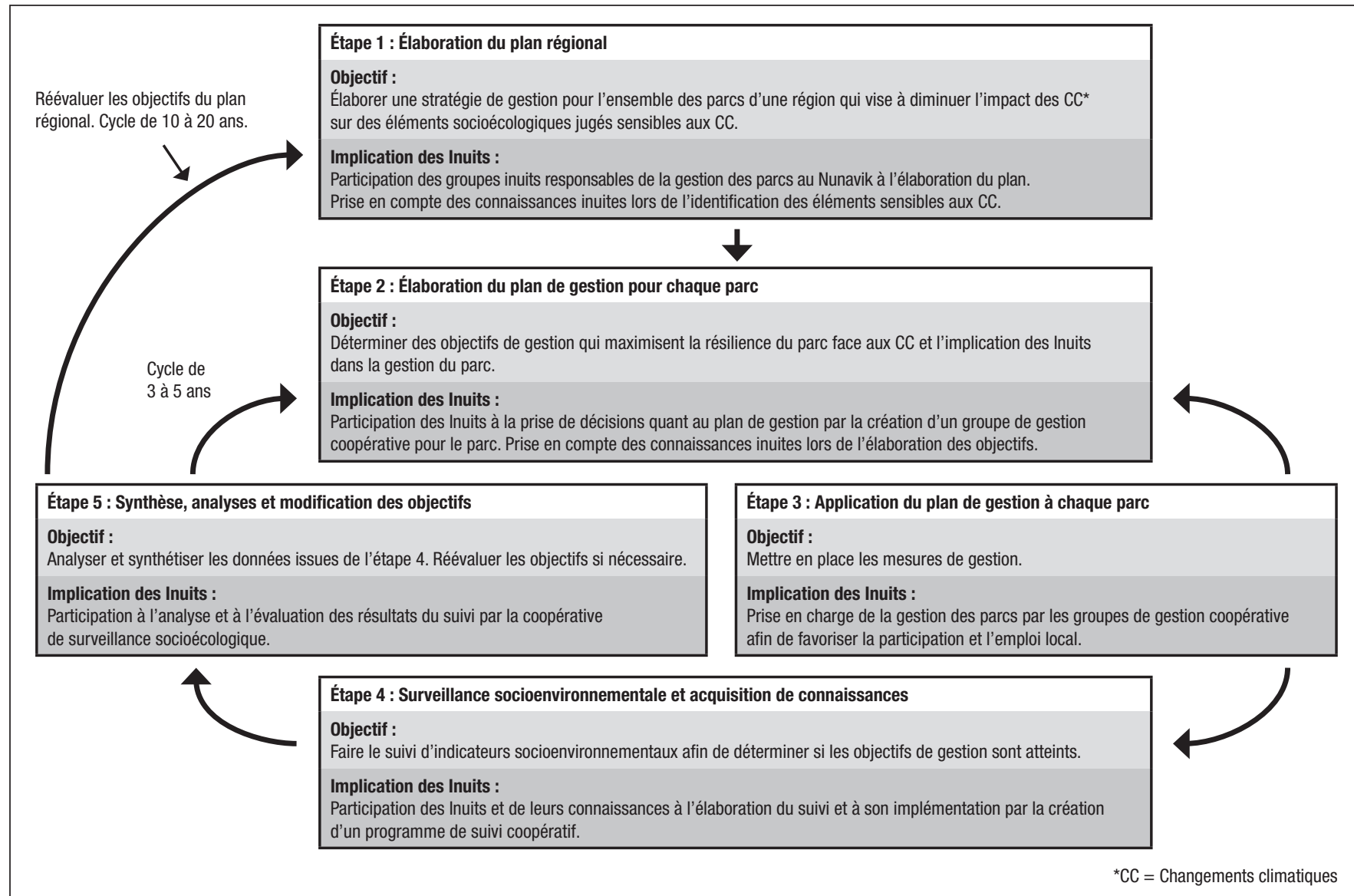

ILLUSTRATION 3 : Schéma du modèle de cogestion adaptative proposé pour les parcs du Nunavik. Pour chaque étape, nous présentons une description de l'objectif et l'implication possible des Inuits (source : compilation des auteurs).

\section{Présentation du modèle}

Étape 1 : Élaboration d'un plan de gestion régional Les changements climatiques auront des conséquences importantes sur les espèces et leurs écosystèmes. Il a été démontré (dans une étude incluant 99 espèces d'oiseaux, insectes et plantes) que, au cours du siècle dernier, les espèces ont migré vers le nord à une vitesse moyenne de $6,1 \mathrm{~km} /$ décennie pour «suivre» leur niche bioclimatique (Parmesan et Yohe, 2003). Au Canada, plus de $50 \%$ des parcs nationaux canadiens devraient subir un changement dans la composition de leur végétation d'ici 2080, ce qui pourrait apporter des modifications aux écosystèmes (Scott et al., 2002). Il apparaît ainsi que l'ampleur des changements en cours dépasse les limites d'un seul parc. Ceci impose de se doter d'un plan de gestion des parcs à l'échelle régionale (par exemple, le nord-est de l'Amérique du Nord), transcendant les juridictions et les limites politiques, et ayant une longue portée temporelle, afin d'anticiper le mieux possible les changements à venir (Hannah, 2010).

L'élaboration d'un plan de gestion régional (par exemple, le nord-est de l'Amérique du Nord) permettra d'identifier les faiblesses du réseau de parcs actuel face aux effets des changements climatiques, et ce, à partir des connaissances actuelles.
L'idée est de prévenir au lieu de guérir, car les actions prises dès aujourd'hui pourront faire économiser temps et argent dans le futur (Hannah et Salm, 2005). Pour ce faire, le plan devra identifier les espèces et les écosystèmes qui semblent les plus sensibles face aux changements et qui devront faire l'objet d'un suivi particulier. Par exemple, certaines espèces qui ne se retrouvent pas dans le nord du Québec présentement pourront s'y retrouver dans le futur si elles sont capables de migrer. Ainsi, un plan régional pourra spécifier qu'il est essentiel de conserver certaines de ces espèces. De plus, le plan servira à évaluer les écosystèmes les plus à risque face aux changements climatiques de sorte que des mesures de gestion concertées seront établies pour les parcs de l'ensemble de la région (la région étant toujours entendue comme un territoire qui transcende les frontières).

Le plan régional devra également avoir une vision à long terme (par exemple 50 ans) (Hannah et Salm, 2005), ce qui permettra d'anticiper sur une période plus longue les changements prévus pour certains éléments identifiés comme sensibles dans le plan régional. Il devra également être flexible et évoluer dans le temps en fonction de l'acquisition de nouvelles connaissances. Nous croyons que cette étape du modèle devra être révisée tous les 10 à 20 ans (voir illustration 3). 
L'élaboration du plan régional fera intervenir les connaissances scientifiques, mais aussi les connaissances locales et traditionnelles des Inuits, documentées par les groupes de travail ci-mentionnés ou lors d'études indépendantes. La combinaison de ces différents savoirs permettra d'obtenir des informations à différentes échelles sur les changements à considérer et d'intégrer les perspectives et préoccupations des Inuits. Par exemple, les connaissances par rapport aux territoires nordiques que possèdent les Inuits pourront servir à identifier des corridors potentiels à maintenir pour favoriser la connectivité entre les différents parcs du Nunavik et le reste du territoire concerné par le plan régional.

Il serait primordial que les membres de l'ARK, actuels responsables de la gestion des parcs au Nunavik, participent à l'élaboration du plan régional, un plan qui est défini en fonction d'impératifs qui débordent, comme on l'a dit plus haut, les frontières nationales. Leur rôle ne serait pas seulement consultatif, mais aussi décisionnel (par exemple dans le domaine des mesures à mettre en place). Idéalement, il serait intéressant que les membres des comités d'harmonisation mis sur pied pour le développement et la gestion des parcs au Nunavik soient aussi présents. Les comités d'harmonisation, par exemple, sont mis sur pied pour chaque parc du Nunavik. Selon le gouvernement du Québec, ces comités ont pour mandat d'associer le milieu régional au développement et à la gestion des parcs. Ces comités sont composés, entre autres, des élus municipaux et de représentants de la Société Makivik. Leur présence pourrait non seulement faciliter la représentation des intérêts locaux dans l'élaboration du plan régional, mais aussi favoriser le transfert d'information depuis et vers les communautés locales.

\section{Étape 2 : Élaboration du plan de gestion pour chaque parc}

En fonction du plan régional, un plan de gestion devra être élaboré pour chaque parc. Les objectifs du plan de gestion différeront s'il s'agit d'un parc déjà établi ou d'un parc en devenir. Dans le premier cas, le nouveau plan de gestion sera contraint par le plan original qui n'était pas nécessairement conçu pour tenir compte des changements climatiques. Dans le cas d'un nouveau parc, le plan pourra intégrer les changements climatiques en tant que thème central autour duquel le reste du plan sera établi (Dudley et al., 2010). Dans les deux cas, le plan de gestion devra aussi inclure des objectifs liés à la cogestion (un objectif de cogestion pourrait être, par exemple, de mettre en place un mode de transition vers une gestion plus coopérative du parc, au sein de laquelle les comités d'harmonisation pourraient obtenir de véritables pouvoirs décisionnels). Comme le plan régional, le plan de gestion pour chaque parc devra avoir une vision à long terme, c'est-à-dire sur 50 ans au lieu des 5 à 10 ans habituels (Hannah et Salm, 2005). Les objectifs du plan de gestion du parc devront toutefois être révisés tous les 3 à 5 ans (voir illustration 2).

Un des principaux objectifs d'un parc est de maintenir, voire de restaurer la biodiversité à l'intérieur de ses limites. Cet objectif demeurera dans le contexte des changements climatiques, car il a été démontré qu'une biodiversité élevée permet aux écosystèmes d'être plus résilients face aux changements (Chapin et al., 2000). Un autre objectif des parcs à prendre en considération est les biens et services écologiques qu'ils procurent (Dudley et al., 2010). Enfin, un des rôles importants des parcs du Nunavik, selon la perspective des organisations inuites, est la stimulation du tourisme et le développement économique des communautés (ARK, 2007; Société Makivik, 2007).

Ainsi, le plan de gestion d'un parc devra inévitablement viser à maintenir et à augmenter la résilience des écosys tèmes qu'il comprend (à travers le maintien et la restauration de la biodiversité), les biens et services écologiques qu'il procure, tout en offrant des opportunités de développement pour les membres des communautés locales (Hansen et al., 2003). Voici cinq points sur lesquels il sera important de se pencher pour déterminer les objectifs de gestion de chaque parc si l'on veut maintenir ces rôles à long terme :

1- Identifier les stress, autres que les changements climatiques (par exemple : perte d'habitat, pollution, surexploitation, etc.), qui menacent la diversité des espèces et des écosystèmes du parc;

2- Déterminer les éléments de la biodiversité qui sont le plus à risque face aux changements climatiques;

3- Déterminer parmi les biens et services écologiques que procure le parc ceux qui sont le plus à risque face aux changements climatiques;

4- Identifier le type de menace climatique qui risque d'avoir le plus d'effets négatifs sur l'ensemble d'un parc (par exemple : sécheresses, pluies, événements climatiques extrêmes, etc.);

5- Évaluer l'impact potentiel des changements climatiques sur le mode de vie local, le tourisme et le développement socioéconomique.

Les connaissances des Inuits devront évidemment être prises en compte lors de l'évaluation de chacun de ces points, par la participation des groupes de travail sur le savoir traditionnel.

Pour les parcs en devenir, on tentera de remédier aux points faibles du réseau existant tels qu'identifiés à l'étape 1 lors de la mise en place des nouveaux parcs (Hannah et Salm, 2005). Toutefois, nous savons que les décisions d'implantation de nouveaux parcs au Nunavik sont influencées par les intérêts de la société énergétique Hydro-Québec et des compagnies minières de la région, ce qui peut limiter les options quand il viendra temps de remédier aux points faibles du réseau existant.

Il est actuellement proposé que les plans de gestion des parcs, dans le contexte des changements climatiques, puissent permettre aux limites de ces derniers d'être dynamiques dans le temps (Hannah et al., 2007). En effet, une limite dynamique permettra aux espèces et écosystèmes qui sont forcés de se déplacer à cause des changements climatiques d'avoir un refuge au fil du temps (Hannah et al., 2007). L'élaboration d'un plan de gestion incorporant cette nouvelle approche apporterait certes de nouveaux défis pour les parcs du Nunavik, mais serait très novatrice et pourrait servir à atténuer certains impacts non anticipés des changements climatiques. L'élaboration du plan de gestion est une étape cruciale de la gestion des parcs. Pour cette étape, il existe déjà une façon de 
faire au Nunavik qui implique les Inuits. En effet, ces derniers sont représentés lors de l'élaboration des plans de gestion par l'ARK et par les groupes de travail et comités d'harmonisation mis sur pied par cette dernière. Selon notre modèle de cogestion adaptative, le plan de gestion de chaque parc devrait être fréquemment réévalué et bonifié (si nécessaire), de même que la manière dont les acteurs locaux sont appelés à y participer. Des mécanismes devraient ainsi être mis en place pour assurer le suivi. Un tel mécanisme pourrait passer par l'établissement d'une coopérative de suivi environnemental dont nous parlerons à l'étape 4.

\section{Étape 3 : Application du plan de gestion à chaque parc}

Les outils de gestion utilisés pour réduire la vulnérabilité des espèces et des écosystèmes (dont ceux des parcs) face aux changements climatiques sont les mêmes que ceux déjà utilisés en conservation (par exemple : maintenir ou augmenter les populations, favoriser la connectivité, réduire les stresses existants, etc.) (Mawdsley et al., 2009; Steffen et al., 2009). Par contre, dans le contexte des changements climatiques, leur application devra être plus agressive, proactive et innovatrice (Hannah et Salm, 2005). À titre d'exemple, certaines espèces utilisent les ponts de glace pour se déplacer du continent vers les îles (Lomolino, 1986). Si les ponts de glace ne se forment plus, une méthode dite agressive pourrait être de transporter, au besoin, des individus de ces espèces du continent vers les îles afin d'assurer le maintien des populations insulaires et de leur diversité génétique.

Nous suggérons que la mise en place des mesures de cogestion soit prise en charge par des organismes locaux. À ce niveau, les parcs du Nunavik sont déjà sur la bonne voie, puisque le mandat de la gestion des parcs est confié à l'ARK, guidée par les comités d'harmonisation. Toutefois, l'intégration des Inuits, comme dans le cas de l'élaboration du plan de gestion (étape 2), pourrait être bonifiée par des comités de gestion coopérative des parcs. Une bonne communication serait nécessaire entre ces comités de gestion et les communautés locales afin de s'assurer que les intérêts et opinions de ces dernières soient pris en compte au cours de cette étape.

\section{Étape 4 : Surveillance socioenvironnementale et acquisition de connaissances}

La surveillance socioenvironnementale et l'acquisition de connaissances sont essentielles dans un processus de cogestion adaptative. Elles permettent de quantifier les résultats des «expériences de gestion» pour ensuite, si nécessaire, les améliorer (Welch, 2005). La surveillance socioenvironnementale et l'acquisition de connaissances qui en découleront seront structurées en fonction des objectifs qui auront été fixés aux étapes 1 et 2 . La surveillance socioenvironnementale consiste à faire un suivi à long terme de certains indicateurs préalablement identifiés par les gestionnaires afin d'évaluer les mesures de gestion. Dans un mode de gestion adaptative conventionnel, l'approche scientifique est normalement utilisée pour la prise de données (Welch, 2005). Toutefois, dans un modèle de cogestion, la science doit être associée aux connaissances traditionnelles et locales.
Pour cette étape, nous suggérons donc une participation réelle et continue des membres des communautés inuites au suivi socioenvironnemental des parcs. Le cas de l'Arctic Borderlands Ecological Knowledge Coop (ABEKC) offre un exemple intéressant (Kofinas et al., 2002; Eamer, 2006). L'ABEKC est né d'une rencontre entre des leaders autochtones, des agents gouvernementaux, des membres de communautés autochtones et des scientifiques dont le but était de trouver une manière d'accroître le suivi environnemental dans l'aire de répartition de la harde de caribous de la rivière Porcupine, qui se déplace entre les Territoires du Nord-Ouest, le Yukon et l'Alaska. Depuis 1999, l'ABEKC fait un suivi environnemental de l'aire de répartition de la harde basé sur le savoir de chasseurs locaux combiné à des indicateurs de changements basés sur des programmes scientifiques (par exemple : données climatiques, estimés d'abondance des caribous, nombre de touristes visitant les communautés, etc.). Le programme de l'ABEKC diffère de la plupart des études sur le savoir traditionnel et local du fait qu'il n'est pas basé sur des entrevues en profondeur avec des aînés. Le programme de l'ABEKC est plutôt basé sur des entrevues annuelles structurées, «conduites par des membres des communautés et qui mettent l'emphase sur ce que les chasseurs, pêcheurs et cueilleurs les plus actifs des communautés ont observé au cours de l'année précédant l'entrevue»(Eamer, 2006, TLDA). L'ABEKC est aussi géré de manière coopérative, et chaque décision importante est prise par consensus lors des rencontres annuelles, auxquelles assistent les membres des organisations impliquées.

Un modèle semblable à celui de l'ABEKC, mais qui tiendrait compte des limites de ce dernier (par exemple : manque de personnel qualifié pour analyser les données, difficultés dans la gestion de la base de données), pourrait être mis en place pour les parcs du Nunavik. Une telle coopérative de suivi socioenvironnemental pourrait aider à construire des ponts entre les différents niveaux d'organisation impliqués dans la création et la gestion des parcs. Une telle coopérative pourrait potentiellement contribuer à développer des liens de confiance entre les membres de différents partis impliqués, un aspect non négligeable pour le succès des entreprises de cogestion (Berkes, 2009).

\section{Étape 5 : Synthèse, analyses et modification des objectifs}

La synthèse, analyses et modification des objectifs, consiste à faire l'analyse des données de suivi de la surveillance socioenvironnementale. Dans un mode de cogestion adaptative, cette analyse des données de suivi doit se faire sur une base régulière (par exemple, 3 à 5 ans) afin de pouvoir réajuster promptement les mesures de gestion lorsque nécessaire. Les résultats doivent être diffusés aux groupes concernés sur une base régulière, et ces derniers doivent également être impliqués si des changements dans les objectifs de gestion sont prévus. Ceci est une étape très importante de la cogestion adaptative qui doit impérativement être respectée si on ne veut pas que la cogestion prenne la forme d'une simple consultation, mais que l'implication des Inuits soit continue et significative. 


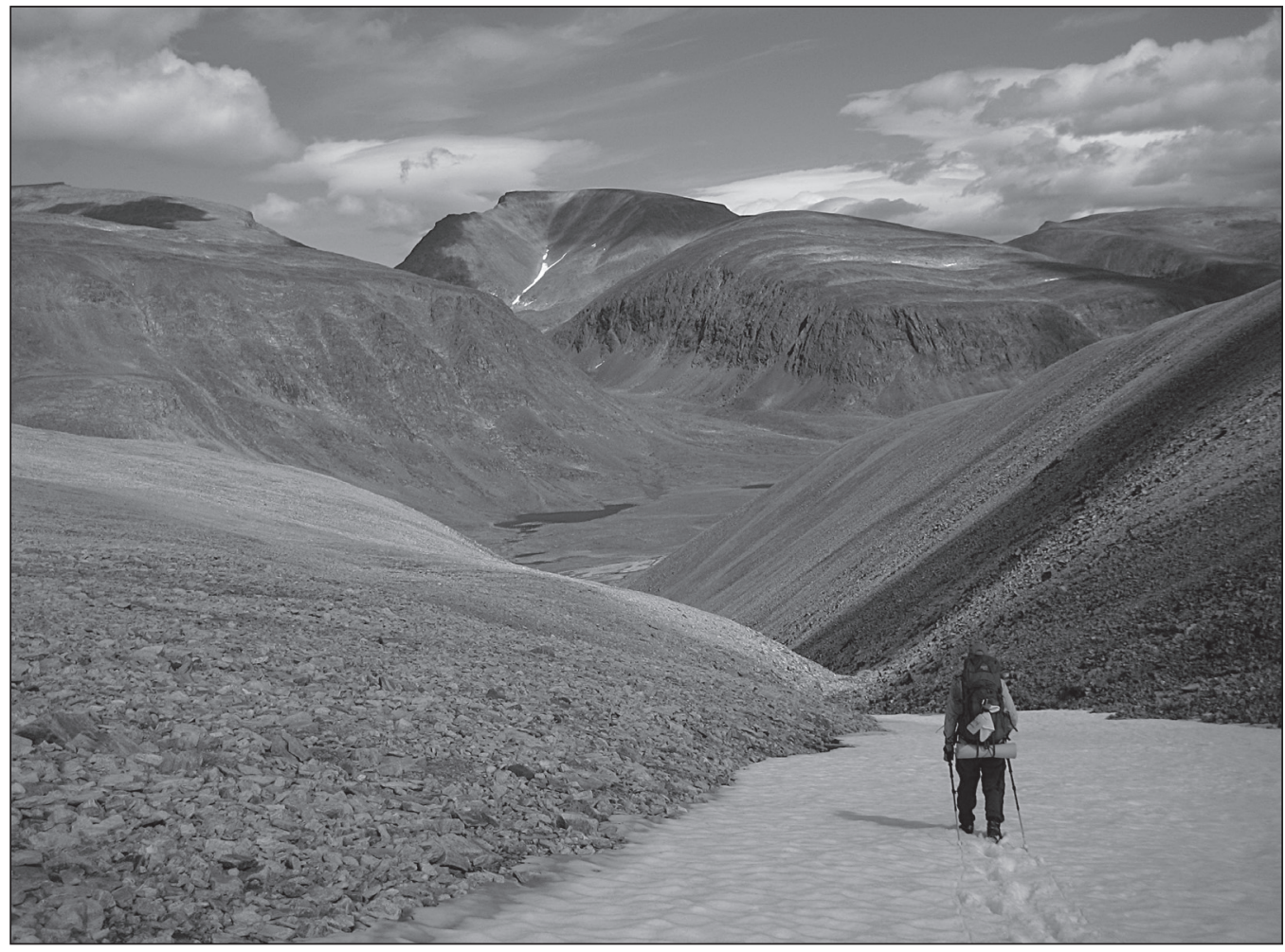

\section{ILLUSTRATION 4 :}

Point de vue sur le futur parc national de la Kuururjuaq (photo : Stéphane Cossette, gracieuseté de l'Administration régionale Kativik (ARK)).
Telle que mentionnée à l'étape 4, la mise en place d'une coopérative de suivi socioenvironnemental pourrait servir de forum pour diffuser l'information obtenue lors du suivi et favoriserait l'implication des résidants à l'élaboration des nouveaux objectifs de gestion, si nécessaire. Ainsi, en plus de contribuer à l'acquisition de connaissances et au suivi de l'état des parcs gérés, une telle coopérative pourrait organiser des rencontres annuelles regroupant tous les groupes impliqués de près ou loin dans la gestion et le développement des parcs du Nunavik. Ces groupes de participants se trouveraient alors en situation d'apprentissage face aux mesures de gestion posées dans le passé (pour plus d'information sur l'apprentissage social et la cogestion, voir Armitage et al., 2008). Ultimement, il incomberait au groupe responsable du développement des mesures de gestion de prendre les décisions nécessaires pour modifier et mettre en place les nouvelles mesures. La boucle de la cogestion adaptative recommencerait alors à l'étape 2 , et ainsi de suite pour les décennies à venir (voir illustration 2).

\section{Discussion}

\section{Principaux défis liés au modèle de cogestion adaptative proposé}

La nécessité de mettre en place le modèle de cogestion adaptative que nous proposons est inspirée de deux idées. Tout d'abord, les changements climatiques représentent, selon nous, un des stress les plus importants pour les écosystèmes nordiques. Pour cette raison, nous croyons qu'ils devraient être davantage considérés lors de l'élaboration des mesures de gestion des parcs. Aussi, dans une optique de développement durable et de démocratisation de la gestion, nous adoptons le point de vue de Carlsson et Berkes (2005), selon lequel le partage du pouvoir devrait être vu comme le résultat de la cogestion, et non pas comme son point de départ. Pour ce faire, la cogestion doit être considérée comme un "processus d'apprentissage par problèmes (au lieu d'un arrangement statique)» (Berkes, 2009, TLDA). Cela justifie notre proposition d'un modèle de cogestion adaptable au fil du temps. Évidemment, nous sommes conscients que la mise en place d'un tel modèle ne pourrait se faire sans embûches. Nous élaborons ici sur les principaux questionnements et défis anticipés.

L'un des premiers défis pour la mise en place de notre modèle de cogestion adaptative est lié à l'urgence d'agir. En effet, les conséquences des changements climatiques sur les parcs et les systèmes socioécologiques se font déjà sentir et, selon les modèles climatiques, la tendance devrait s'accentuer dans les prochaines années (IPCC, 2007). Ainsi, le modèle que nous proposons devrait être mis en place rapidement. Pour ce faire, le ministère du Développement durable, de l'Environnement et des Parcs, qui est l'autorité responsable des parcs au Québec, devrait définir dans un court délai (idéalement d'ici 5 ans) sa politique quant à la gestion des parcs dans le contexte des changements climatiques. Ce travail devrait se faire en collaboration avec les organisations de cogestion. Lorsque le ministère aura défini sa politique dans ce domaine, Parcs Nunavik pourra participer à l'élaboration du plan régional dans lequel le Nunavik se situe et procéder à l'élaboration d'un plan de gestion pour chaque parc. De plus, Parcs Nunavik devra tenir compte des changements liés aux tourismes dans cette région. Traditionnellement, le 
tourisme de cette région est principalement lié aux activités de pourvoiries, mais cette situation pourrait changer avec le Plan Nord et la création de nouveaux parcs (Québec, 2012). En effet, le tourisme de villégiature pourrait augmenter et par le fait même augmenter la pression sur les parcs (Stewart et al., 2005; Québec, 2012).

Un second défi du modèle proposé est lié à la difficulté de déterminer les conséquences et opportunités que représenteront les changements climatiques pour chaque parc. Cette difficulté émerge du fait que, actuellement, les études sur les impacts qu'auront les changements climatiques sont basées sur des connaissances scientifiques qui décrivent ce phénomène à grande échelle (Parmesan et Yohe, 2003). C'est entre autres pour cette raison qu'il est nécessaire d'utiliser toutes les sources de connaissances disponibles sur l'état actuel des systèmes socioécologiques et sur les changements qu'ils subissent. Au Nunavik, les connaissances des Inuits peuvent contribuer à documenter ce phénomène. Or, combiner différents types de savoirs n'est pas une tâche facile, particulièrement lorsqu'on veut combiner des connaissances fondées sur différentes visions du monde (par exemple : connaissances scientifiques versus connaissances autochtones) (Reid et al., 2006). Le succès d'une telle opération requiert souvent le développement de liens de confiance et de respect entre les détenteurs de chaque type de savoir. À cet égard, le modèle de cogestion adaptative que nous proposons a le potentiel de servir de catalyseur facilitant l'établissement de ces liens.

Pour le troisième défi, il faut considérer que la mise en place de mesures de gestion visant à atténuer l'impact des changements climatiques augmentera les besoins en ressources humaines et les coûts de gestion des parcs (Welch, 2005). De la même façon, la mise en place de groupes de gestion et de surveillance socioenvironnementale coopératifs nécessiterait des ressources humaines et financières additionnelles. Les coûts de transport, par exemple, liés à la tenue de rencontres dans le Nord sont considérables. Aussi, notre expérience nous a montré que, dans les communautés nordiques peu populeuses, les leaders locaux sont souvent surchargés de travail du fait de leur participation à un nombre important de différents comités.

Pour ce qui est des ressources financières, une mobilisation des populations locales aiderait à inciter la classe politique à investir plus d'argent et de ressources dans la gestion des parcs (Welch, 2005). D’ailleurs, l'ARK mentionnait la nécessité que des ressources humaines et financières adéquates soient disponibles pour que le projet de parc national de la Kuururjuaq soit une réussite (ARK, 2007) (voir illustration 4). Sur ce point, le modèle de cogestion adaptative pourrait servir de levier. Effectivement, le poids politique de plusieurs groupes d'intérêts représentant une vision commune a de fortes chances de favoriser l'investissement financier et humain de la part de partenaires (gouvernementaux ou non) pouvant fournir ces ressources. Pour cela, il faudrait cependant que l'ébauche du modèle soit d'abord mise en place.

En ce qui a trait aux ressources humaines, nous reconnaissons que notre modèle pourrait initialement ajouter une charge de travail sur les leaders locaux. À moyen terme toutefois, un tel modèle de cogestion devrait inclure des mécanismes visant à favoriser le développement du leadership et de la capacité de gestion chez d'autres acteurs locaux (par exemple en essayant de stimuler la participation des jeunes aux groupes de travail et comités de cogestion). Favoriser la participation de nouveaux acteurs locaux contribuerait à stimuler le développement socioéconomique des communautés, à réduire la surcharge de travail de certains leaders et à augmenter la représentativité des groupes de cogestion.

La question de la représentativité des groupes de cogestion est un des grands défis de la cogestion. À ce sujet, la cogestion adaptative, comme la cogestion, n'offre pas de garantie contre la gestion inéquitable des ressources. Dans certains cas, malgré le processus participatif, seuls les intérêts de quelques groupes sont mis de l'avant. Une solution à ce défi serait la création de groupes de cogestion incluant une diversité de représentants au niveau local (non seulement les élus municipaux) et une diversité d'organisations au niveau régional, comme les organismes touristiques.

\section{Conclusion}

Nous reconnaissons ici que l'intérêt que nous portons à la cause des changements climatiques est influencé par notre domaine d'étude : la biologie. Ainsi, bien que nous mettions les changements climatiques à l'avant-plan de notre modèle, nous sommes conscients que cette problématique puisse être de moindre importance pour certains groupes impliqués dans la cogestion des parcs. Puisque l'objectif de la cogestion est de représenter les intérêts des groupes impliqués, il serait donc possible que la place centrale qu'occupent les changements climatiques soit revue au fil du temps.

Dans les faits, il n'existe pas un type de gouvernance idéal, applicable à toutes les situations de gestion environnementale (Ostrom et al., 2007). De même, il n'existe pas de solution idéale, applicable à tous les défis de la cogestion (Berkes, 2009). Chaque contexte de cogestion est unique (même à l'intérieur d'une même région), avec ses propres forces et faiblesses, et sa diversité d'intérêts locaux et régionaux. Ainsi, la mise en place de notre modèle et les solutions aux défis qu'il engendrera dépendront de ce contexte spécifique. L'expérimentation est encore nécessaire dans le domaine de la cogestion (Ostrom et al., 2007). À ce titre, nous voyons notre modèle non pas comme un projet normatif à appliquer tel quel, mais plutôt comme un moyen de stimuler la réflexion. Il restera donc aux acteurs concernés à voir s'il leur convient et à adapter sa mise en application, si nécessaire.

\section{Références}

ARK — Administration régionale Kativik (2007) Mémoire présenté aux audiences publiques sur le projet de parc national de la Kuururjuaq, Kangiqsualujjuaq : Administration régionale Kativik. 7 p. ARMITAGE, Derek; Fikret BERKES et Nancy DOUBLEDAY (dir) (2007a) "Introduction: moving beyond co-management ", DANS Adaptive comanagement: collaboration, learning, and multi-level governance, p. 1-15. UBC Press : Vancouver, BC.

ARMITAGE, Derek; Fikret BERKES et Nancy DOUBLEDAY (dir.) (2007b) Adaptive co-management: collaboration, learning, and multi-level governance, Vancouver, BC : UBC Press BC. 344 p. 
ARMITAGE, Derek; Melissa MARSCHKE et Ryan PLUMMER (2008) "Adaptive co-management and the paradox of learning», Global Environmental Change, vol. $18, \mathrm{n}^{\circ} 1$, p. 86-98.

BERKES, Fikret (1999) Sacred ecology: traditional ecological knowledge and resource management, Philadelphia : Taylor \& Francis. 209 p.

BERKES, Fikret (2009) «Evolution of co-management: Role of knowledge generation, bridging organizations and social learning», Journal of Environmental Management, vol. 90, nº 5, p. 1692-1702.

BERKES, Fikret; Derek ARMITAGE et Nancy DOUBLEDAY (dir.) (2007) "Synthesis: adapting, innovating, evolving», DANS Adaptive co-management: collaboration, learning, and multi-level governance, Vancouver : UBC Press. p. 308-327.

BERKES, Fikret et Carl FOLKE (1998) Linking social and ecological systems : management practices and social mechanisms for building resilience, Cambridge : Cambridge University Press. 459 p.

BERKES, Fikret; P. J. GEORGE et R. J. PRESTON (1991) «Co-management: The evolution in theory and practice of the joint administration of living resources", Alternatives, vol. 18, n 2, p. 12-18.

BORRINI-FEYERABEND, Grazia; Michel PIMBERT; M. Taghi FARVAR; Ashish KOTHARI et Yves RENARD (2004) Sharing power: learningby-doing in co-management of natural resources throughout the world, Cenesta, Tehran : IIED and IUCN/CEESP/CMWG. 456 p.

CALLAGHAN, Terry V.; Lars Olof BJORN; Yuri CHERNOV; Terry CHAPIN; Torben R. CHRISTENSEN; Brian HUNTLEY; Rolf A. IMS; Margareta JOHANSSON; Dyanna JOLLY; Sven JONASSON; Nadya MATVEYEVA; Nicolai PANIKOV; Walter OECHEL; Gus SHAVER; Sibyll SCHAPHOFF; Steohen SITCH et Christoph ZOCKLER (2004) «Synthesis of effects in four Arctic subregions", Ambio, vol. 33, n ${ }^{\circ}$, p. 469-473.

CARLSSON, Lars et Fikret BERKES (2005) «Co-management: concepts and methodological implications", Journal of Environmental Management, vol. $75, \mathrm{n}^{\circ}$ 1, p. 65-76.

CHAMBERS, Jennifer; Yanick GENDREAU; Marie Claire BÉDARD; A. E. GAGNON et Fanie PELLETIER (2010) Impacts des changements climatiques sur la biodiversité du Québec : Aires protégées, Québec : Ministère du Développement durable, de l'Environnement et des Parcs. 75 p.

CHAPIN, F. Stuart; Erika S. ZAVALETA; Valerie T. EVINER; Rosamond L. NAYLOR; Peter M. VITOUSEK; Heather L. REYNOLDS; David U. HOOPER; Sandra LAVOREL; Osvaldo E. SALA; Sarah E. HOBBIE; Michelle C. MACK : et Sandra DÍAZ (2000) "Consequences of changing biodiversity", Nature, vol. 405, n 6783, p. 234-242.

DUDLEY, Nigel; Sue STOLTON; Alexander BELOKUROV; Kathy MACKINNON; Trevor SANDWITH et Nik SEKHRAN (2010) Natural solutions: Protected areas helping people cope with climate change, Gland (Switzerland),Washington DC et New York : IUCN-WCPA, TNC, UNDP, WCS, The World Bank, WWF. 130 p.

EAMER, Joan (2006) « Keep it simple and be relevant: the first ten years of the Arctic Borderlands Ecological Knowledge Co-op», DANS REID, Walter V.; Fikret BERKES; T. WILBANKS et Doris CAPISTRANO (sous la direction de), Bridging scales and knowledge systems: concepts and applications in ecosystem assessment, p. 185-206. Washington, D.C : Island Press.

EM - Évaluation des écosystèmes pour le millénaire (2005) Millennium Ecosystem Assessment - Ecosystems and Human Well-being: Biodiversity Synthesis, Washington, DC. : World Resources Institute. 100 p.

FOLKE, Carl; Steve CARPENTER; Thomas ELMQVIST; Lance GUNDERSON; Crawford Stanley HOLLING; Brian WALKER;
Jan BENGTSSON; Fikret BERKES; Johan COLDING; Kjell DANELL; Malin FALKENMARK; Line GORDON; Roger KASPERSON; Nils KAUTSKY; Ann KINZIG; Simon LEVIN; Karl-Göran MÄLER; Fredrik MOBERG; Leif OHLSSON; Per OLSSON; Elinor OSTROM; Walter REID; Johan ROCKSTRÖM; Hubert SAVENIJE et Uno SVEDIN (2002) Resilience and sustainable development: building adaptive capacity in a world of transformation, Scientific background paper on resilience for the process of The World Summit on Sustainable Development on behalf of the Environmental Advisory Council to the Swedish Government. $34 \mathrm{p}$.

GAGNON, Catherine-Alexandra et Dominique BERTEAUX (2006)

«Integrating traditional and scientific knowledge: management of Canada's national parks», DANS R. RIEWE et J. OAKES (sous la direction de), Climate change: integrating traditional and scientific knowledge, p. 209221. Winnipeg : Aboriginal Issues Press.

GAGNON, Catherine-Alexandra et Dominique BERTEAUX (2009) «Integrating traditional ecological knowledge and ecological science: a question of scale», Ecology and Society, vol. 14, n 2, p. 19.

HANNAH, Lee (2010) «A Global Conservation System for Climate-Change Adaptation", Conservation Biology, vol. 24, n 1, p. 70-77.

HANNAH, Lee; Guy MIDGLEY; Sandy ANDELMAN; Miguel ARAÙO; Greg HUGHES; Enrique MARTINEZ-MEYER; Richard PEARSON et Paul WILLIAMS (2007) «Protected area needs in a changing climate», Frontiers in Ecology and the Environment, vol. 5, n 3, p. 131-138.

HANNAH, Lee et Rod SALM (2005) «Protected areas management in a changing climate», DANS Thomas E. LOVEJOY et Lee HANNAH (sous la direction de), Climate change and biodiversity, p. 418. New Haven et London : Yale University Press.

HANSEN, Lara J.; Jennifer L. BIRINGER et J. R. HOFFMAN (2003) Buying time: a user's manual for building resistance and resilience to climate change in natural systems, World Wildlife Fund. 246 p.

HILBORN, Ray et Donald LUDWIG (1993) «The Limits of Applied Ecological Research», Ecological Applications, vol. 3, nº 4, p. 550-552.

HOLLING, Crawford Stanley (1978) Adaptive environmental assessment and management, London : Wiley. 377 p.

HOLLING, Crawford Stanley (1996) «Surprise for science, resilience for ecosystems, and incentives for people», Ecological Applications, vol. 6, $\mathrm{n}^{\circ} 3$, p. 733-735.

HUNTINGTON, Henry; Terry CALLAGHAN; Shari FOX et Igor KRUPNIK (2004) «Matching traditional and scientific observations to detect environmental change: A discussion on Arctic terrestrial ecosystems», Ambio, Special report, p. 18-23.

IPCC (2007) Impacts, adaptation and vulnerability: contribution of Working Group II to the fourth assessment report of the Intergovernmental Panel on Climate Change, Cambridge, New York : Cambridge University Press. $976 \mathrm{p}$.

KOFINAS, Gary; AKLAVIK; ARCTIC VILLAGE; OLD CROW et FORT MCPHERSON (2002) «Community Contributions to Ecological Monitoring: Knowledge Co-production in the U.S.-Canada Arctic Borderlands», DANS KRUPNIK, Igor et Dyanna JOLLY (sous la direction de), The Earth is Faster Now: Indigenous Observations of Arctic Environmental Change, p. 55-91. Fairbanks : Arctic Research Consortium of the United States.

KREBS, Charles J. et Dominique BERTEAUX (2006) «Problems and pitfalls in relating climate variability to population dynamics», Climate Research, vol. 32, n' 2, p. 143-149.

KRUPNIK, Igor et Dyanna JOLLY (dir.) (2002) The Earth is Faster Now: Indigenous Observations of Arctic Environmental Change. Fairbanks : Arctic Research Consortium of the United StatesFairbanks. 384 p. 
LOMOLINO, Mark V. (1986) «Mammalian community structure on islands: the importance of immigration, extinction and interactive effects », Biological Journal of the Linnean Society, vol. 28, $\mathrm{n}^{\circ} 1-2, \mathrm{p} .1-21$.

MAWDSLEY, Jonnathan R.; Robin O’MALLEY et Dennis S. OJIMA (2009)

"A Review of Climate-Change Adaptation Strategies for Wildlife Management and Biodiversity Conservation », Conservation Biology, vol. 23, no 5, p. 1080-1089.

MCCARTHY, Michael A. et Hugh P. POSSINGHAM (2007) «Active Adaptive Management for Conservation», Conservation Biology, vol. 21, $\mathrm{n}^{\circ} 4$, p. 956-963.

NADASDY, Paul (1999) «The politics of TEK: power and the 'integration' of knowledge», Arctic Anthropology, vol. 36, no 1-2, p. 1-18.

NADASDY, Paul (2003) «Reevaluating the co-management success story», Arctic, vol. 56, n 4 , p. 367-380.

OSTROM, Elinor; Marco A. JANSSEN et John M. ANDERIES (2007) "Going beyond panaceas", Proceedings of the National Academy of Sciences of the United States of America, vol. 104, n 39, p. 15176-15178.

PARMESAN, Camille et Garry YOHE (2003) «A globally coherent fingerprint of climate change impacts across natural systems ", Nature, vol. 421, n 6918, p. 37-42.

PLUMMER, Ryan et Derek ARMITAGE (2007) «A resilience-based framework for evaluating adaptive co-management: Linking ecology, economics and society in a complex world ", Ecological Economics, vol. $61, \mathrm{n}^{\circ} 1$, p. 62-74.

PLUMMER, Ryan et John FITZGIBBON (2004) «Co-management of natural resources: a proposed framework», Environmental Management, vol. $33, \mathrm{n}^{\circ} 6$, p. $876-885$.

PRÉVOT-JULLIARD, Anne Croline; Virginie MARIS; Karine ALAIN et Yves COPPENS (dir.) (2010) Biodiversité : Nouveaux regards sur le vivant, Paris : Le Cherche Midi. 276 p.

QUÉBEC, Ministère du tourisme (2012) Plan de développement de l'industrie touristique 2012-2020 : Un itinéraire vers la croissance, Québec : Ministère du Tourisme. $110 \mathrm{p}$.

REID, Walter. V.; Fikret BERKES; Thomas WILBANKS et

Doris CAPISTRANO (dir.) (2006) Bridging scales and knowledge systems: concepts and applications in ecosystem assessment, Washington, D.C. : Island Press Washington. 343 p.
RIEDLINGER, Dyanna et Fikret BERKES (2001) «Contributions of traditional knowledge to understanding climate change in the Canadian Arctic», Polar record, vol. 37, $\mathrm{n}^{\circ}$ 203, p. 315-328.

SAINSBURY, Keith J.; André E. PUNT et Anthony D. M. SMITH (2000) «Design of operational management strategies for achieving fishery ecosystem objectives », ICES Journal of Marine Science: Journal du Conseil, vol. $57, \mathrm{n}^{\circ} 3$, p. 731-741.

SCOTT, Daniel; Jay R. MALCOLM et Christopher LEMIEUX (2002) «Climate change and modelled biome representation in Canada's national park system: implications for system planning and park mandates », Global Ecology and Biogeography, vol. 11, nº 6, p. 475-484.

SOCIÉTÉ MAKIVIK (2007) Presentation by Makivik Corporation, Johnny Peters, Vice President et George Berthe, Corporate Secretary. Public Hearings on the proposed «Parc national de la Kuururjuaq». Kangiqsualujjuaq : Société Makivik.

STEFFEN, Will; Andrew A. BURBIDGE; Lesley HUGHES; Roger KITCHING; David LINDENMAYER; Waren MUSGRAVE; Marc STAFFORD SMITH et Patricia WERNER (2009) Australia's biodiversity and climate change, Collingwood, Australia : CSIRO publishing. $236 \mathrm{p}$.

STEWART, E. J.; D. DRAPER et M. E. JOHNSTON (2005) «A Review of Tourism Research in the Polar Regions », Arctic, vol. 58, n 4, p. 383-394.

THOMAS, Chris D.; Alison CAMERON; Rhys E. GREEN; Michelle BAKKENES; Linda J. BEAUMONT; Yvonne C. COLLINGHAM; Barrend F. N. ERASMUS; Marrinez F. DE SIQUEIRA; Alan GRAINGER; Lee HANNAH; Lesley HUGHES; Brian HUNTLEY; Albert S. VAN JAARSVELD; Guy F. MIDGLEY; Lera MILES; Miguel A. ORTEGA-HUERTA; A. Townsend PETERSON; Oliver L. PHILLIPS et Stephen E. WILLIAMS (2004) «Extinction risk from climate change», Nature, vol. 427, n 6970, p. 145-148.

WAGNER, Cheryl L. et Maria FERNANDEZ-GIMENEZ (2008) «Does community-based collaborative resource management increase social capital?», Society \& Natural Resources, vol. 21, n 4, p. 324-344.

WALTERS, Carl J. (1986) Adaptive management of renewable resources, New York, London : Macmillan. 374 p.

WELCH, David (2005) «What should protected area managers do in the face of climate change?», The George Wright forum, vol. 22, n 1, p. 75-93.

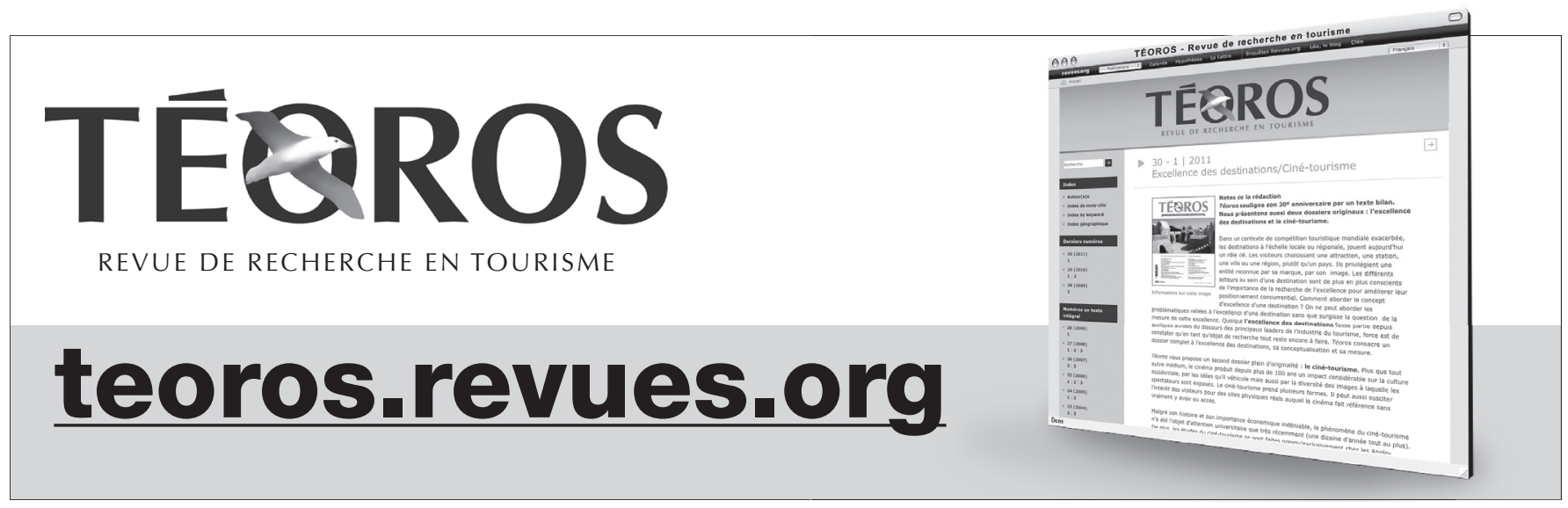

TÉOROS, vol. 31, nº 1, p. 61-71 @ 2012 